\title{
El infinito en la historia de la matemática
}

Claudia Andrea López ${ }^{1}$

\section{Resumen}

Los aspectos históricos del concepto de infinito podrían servir como punto de partida para la comprensión del mismo. El infinito, dentro de la matemática de nivel universitario, ocupa un lugar importante en el currículum de las carreras de Ingeniería. La aclaración de este concepto podría tener entonces consecuencias favorables en el aprendizaje de algunos temas tales como límites, sucesiones, series, área bajo una curva. Y dado que su conceptualización ha sido un proceso largo con muchas dificultades, es preciso analizar tanto la naturaleza de los conceptos involucrados como la génesis del surgimiento. Si bien, el término infinito se utiliza para designar lo que es muy grande, ilimitado o no tiene fin su noción va más allá de lo que nuestra imaginación nos puede llegar a brindar. En este trabajo se destacan los personajes más relevantes a lo largo de la historia que influyeron en la génesis del concepto.

Palabras clave: historia - infinito potencial - infinito actual - personajes destacados.

\section{Abstract}

Historical aspects might constitute a starting point for the understanding of the concept of infinity. Within college-level mathematics, infinity holds an important status in the curriculum of engineering careers. Understanding of this notion may have an important effect on the learning of certain topics such as limits, sequences, series or area of a region under a curve. Since the process which led to the conceptualization of infinity has been a long and difficult one, it is essential to analyze the nature of the concepts involved as well as the genesis of its emergence. Although the term 'infinity' is used to designate something large, unlimited or endless, this notion goes beyond our imagination. This work presents the most important contributors to the development of this concept along history.

Key words: history - potential infinity - actual infinity - relevant personages

1 Universidad de Palermo, Argentina. 



\section{El infinito en la civilización griega}

Históricamente el infinito matemático no puede separarse del aspecto filosófico y religioso, dado que la religión ha tenido una gran influencia en los pensadores y además la matemática estaba estrechamente vinculada con la filosofía. Según Eggers Lan (1995) la filosofía y la matemática se hallaban significativamente interpenetradas.

Al pretender rastrear la historia de una ciencia se puede partir en un comienzo de dos ámbitos distintos: el de la ciencia en particular y el del mundo griego antiguo con su peculiaridad. El historiador germano Kurt von Fritz, citado por Eggers Lan argumenta: "Si la evolución de la matemática griega antigua ha sido -por completo o en forma predominante- de índole exclusivamente matemática, o si han influido en ella ideas filosóficas, es un viejo tema de discusiones. Personalmente me parece evidente -tal como usted piensa- que dicha evolución ha recibido, en distintas épocas, fuertes estímulos por parte de filósofos, aunque va de suyo que también ha habido siempre ulteriores desarrollos de indole exclusivamente matemática".

Así es como las primeras preguntas sobre el infinito surgieron en Grecia al pensar en el espacio y el tiempo, se cuestionaban si serían finitos o infinitos. Por otra parte, además de las dudas existenciales que tenían acerca del mundo se encontraron con otra dificultad: la divisibilidad de la materia. El infinito aparece por primera vez en la civilización griega con Anaximandro, s VI a. c, de la escuela de Tales de Mileto. Anaximandro proponía que la primera sustancia de la cual están hechas todas las cosas es el ápeiron, que concibió como algo neutral, imperecedero, infinito, ilimitado.

Anaximandro comenzó la discusión a favor del infinito: el universo contiene un número infinito de mundos, la duración del universo es infinita, el material del cual están hechas todas las cosas, incluido el agua, es infinito. Lo infinito no tiene en Anaximandro un sentido temporal sino lo más profundo e inabarcable, lo contrario a límite. Simplemente el infinito de Anaximandro es el todo, absolutamente nada hay fuera de él. Posteriormente, el primero que se opuso al infinito fue Aristóteles s. III. a. c, quien cita a Anaximandro en el libro III de Física y trata de refutar su posición. También en Grecia en el s VI a. c surge la figura de Pitágoras y de su escuela. Los pitagóricos pensaban que los números naturales eran la esencia de todas las cosas, y según Aristóteles citado por Rey Pastor \& Babini (1984, pág. 43) fueron los primeros en hacer progresar la matemática y nutridos de ella creyeron que su principio era el de todas las cosas. Como consecuencia de este pensamiento el descubrimiento de pares de cantidades diferentes, los números irracionales, los desconcertó de tal manera que decidieron ocultarlo, prevaleció el secreto ante el descubrimiento. Esto contradecía la creencia de que todo era medible por números y que todas las magnitudes podían expresarse por la razón entre dos números. A partir de este hallazgo cambiaron el rumbo de sus descubrimientos dejaron de 
lado consideraciones aritméticas y algebraicas para darle paso a cuestiones de tipo geométrico. Por eso se ocuparon de reformular toda la teoría para evitar los inconmensurables: "El gran mérito de los pitagóricos fue reformular toda su teoría anterior para evitar encontrarse con los inconmensurables. Así, transformaron su teoría de proporciones en una de transformación de áreas, evitando por poco el desastre" (Díaz García \& Vilela García, 2005, pág. 8) .

Los diferentes problemas que el infinito ocasionaba como consecuencia de las situaciones aparentemente contradictorias caracterizaron al mundo griego en lo que se denominó "horror al infinito". Las paradojas de Zenón de Elea, s V a. c, acerca del infinito y lo infinitesimal lo ubican como un precursor de la Matemática en temas que han sido tratados posteriormente por destacados matemáticos. Por esa época existían dos concepciones opuestas del espacio y el tiempo. Algunos consideraban que el espacio y el tiempo eran indefinidamente divisibles y otros pensaban que el espacio y el tiempo estaban formados por pequeños intervalos indivisibles y en ese caso el movimiento se constituía por una sucesión de pequeños saltos. Se conocen cuatro paradojas de Zenón acerca del movimiento y la continuidad. En la muy conocida paradoja de Aquiles y la tortuga el tiempo y el espacio son infinitamente divisibles. Así es como Aquiles, el más rápido de los hombres no podrá alcanzar nunca al más lento de los animales, la tortuga, si se da a esta una ventaja inicial en la carrera. Pues, mientras Aquiles recorre el camino que la tortuga llevaba por la mencionada ventaja inicial, la tortuga habrá recorrido otra porción, aunque más pequeña. Cuando Aquiles haya llegado a recorrer esta última porción de camino, la tortuga habrá avanzado otra porción más pequeña, y así la tortuga llevará siempre la ventaja en espacios infinitamente pequeños, con lo cual, Aquiles no podrá alcanzarla nunca. En esta paradoja Zenón argumenta contra el movimiento basándose en el supuesto de que el espacio y el tiempo son infinitamente divisibles. Zenón consideraba que el movimiento no existe y que es un engaño de nuestros sentidos, en realidad el problema surge al querer tratar una magnitud continua (el tiempo) como algo discreto. El tema central resultaría ser la aparición de procesos infinitos (infinito potencial) y por eso según Zenón, Aquiles no podría alcanzar la tortuga dado que es imposible realizar una infinidad de actos. Es decir, la suma de un número infinito de intervalos de tiempo positivos no puede ser finita. Esta afirmación de Zenón se contradijo 2000 años más tarde con la creación de la teoría de las series infinitas. Las paradojas de Zenón producían contradicciones al pensar, tanto si se consideraba el espacio y el tiempo como continuos o discontinuos, por eso es que los matemáticos de la época creyeron que la única forma de evitar las dificultades que el infinito ocasionaba era desterrarlo de su ciencia.

Por otra parte, en Atenas, Anaxágoras, s V a. c, intenta refutar esas aporías estableciendo un cierto principio de continuidad, argumentando que no existe lo más pequeño entre lo más pequeño ni lo más grande entre los más grande, sino 
siempre algo aún más pequeño y algo aún más grande. O sea, que toda magnitud puede seguir siendo dividida hacia lo mínimo de modo ilimitado o creciendo hacia lo máximo en un proceso sin fin.

Según Bagni (2001) Anaxágoras es el primer autor recordado en introducir procedimientos infinitesimales, introduce la noción de límite, no en el sentido moderno, sino mediante el llamado método de exhaución griego. Este método se presenta inicialmente en un registro verbal, y posteriormente es puesto en un registro visual por Euclides en el s III a. C, en sus conocidos "Elementos", aunque dicho método es atribuido a Eudoxo s IV a. c. La expresión visual del método les resultó particularmente útil para poder interpretar las construcciones en un gráfico y razonar visualmente.

El desarrollo de la matemática en la Grecia del siglo IV a. c. se caracteriza fundamentalmente por la influencia de dos escuelas filosóficas fundadas en Atenas, la Academia fundada por Platón en el 387 a. c y el Liceo creado por Aristóteles en el 335 a. c.. Los alumnos de la Academia de Platón motivados por su maestro se interesaron en el estudio de las matemáticas, fue un gran inspirador de su época que supo transmitir su entusiasmo por la Matemática. En la Academia se consolida el problema de la inconmensurabilidad como origen de la demostración y es en las obras de Platón que se observa la influencia del descubrimiento de los irracionales, precisamente en uno de sus cuatro Diálogos, "Teeteto", escrito entre 369-368 a. c. Hay cuatro Diálogos de la obra platónica que constituyen un conjunto que comparte la estructura y el significado, escritos en el siguiente orden: Parménides, Teeteto, Sofista y Político, en ellos se observa rasgos de composición distintivos y específicos que difieren del resto de los diálogos platónicos. El "Parménides" y el "Teeteto" tratan de las conversaciones que Sócrates mantiene con sus interlocutores. En el primer diálogo del "Teeteto" aparece la figura de Euclides conversando con Terpsión acerca de su encuentro con Teeteto. Por otra parte, Teeteto es citado en el catálogo eudemio de matemáticos y también en el tratado histórico de Proclo como un matemático reconocido. En particular, los libros VII, VIII, X y XIII de los Elementos de Euclides están inspirados en doctrinas de Teeteto y son parcialmente deudores de su obra. "Así se atribuye a Teeteto de Atenas, inmortalizado en el diálogo de ese nombre, el estudio de los inconmensurables, con lo cual habría sentado las bases de las propiedades que más tarde se reunirán en el Libro X de los Elementos de Euclides" (Rey Pastor \& Babini, 1984, pág. 60).

En este escrito, en el que Sócrates dialoga con Teodoro y Teeteto, se pone de manifiesto la búsqueda de la definición del conocimiento. Ambos eran notables matemáticos que se destacaban por sus investigaciones acerca de los números irracionales. El descubrimiento de los irracionales provocó que Platón abandonara las investigaciones aritméticas para dedicarse a investigaciones geométricas.

Eudoxo (408 a. c) nacido en la ciudad de Cnido, fue el matemático más grande del siglo IV, también perteneció a la Academia de Platón aunque se había formado 
de muy joven con los pitagóricos. A Eudoxo se debe la noción de "tan pequeño como se quiera", antecedente de nuestro proceso de paso al límite. Algunos de los libros de Euclides están basados en la teoría de Eudoxo. De los trece libros de Los Elementos de Euclides el décimo es el más difícil, extenso y el que se ocupa de los irracionales. A Eudoxo se le atribuye, entre otras cosas, el método de exhaución aunque dicho método corresponde en realidad a un teorema, Proposición I del libro $\mathrm{X}$ de Euclides, que expresa que dadas dos magnitudes distintas, si de la mayor se sustrae una magnitud mayor que su mitad, del resto se sustrae una magnitud mayor que su mitad y si este proceso se repite continuamente, quedará alguna magnitud más pequeña que la menor de las magnitudes dadas inicialmente.

Habitualmente este método se usa para considerar el círculo como límite de polígonos regulares cuando el número de lados aumenta hasta el infinito. Sin embargo, ésta no fue la forma en que los griegos utilizaron el teorema sino un argumento de reducción al absurdo para evitar el uso del infinito. Tal como lo expresa Bagni (2001) este resultado tan sencillo tuvo consecuencias importantes para el posterior desarrollo de la matemática, dado que en este principio subyace la formulación de la idea de límite sin hacer uso del infinito.

La figura de Aristóteles resulta importante en la solución al problema del infinito. Según Toledo Prats (2003, pág. 80) Aristóteles intenta refutar las paradojas de Zenón para afianzar los conceptos fundamentales de la matemática. Rechaza la noción del infinito por las contradicciones que ocasionaba, sin embargo trata de resolver las controversias que generaba concibiendo dos formas diferentes del mismo. El infinito como un proceso de crecimiento sin final, o de subdivisión sin final, infinito potencial, y el infinito como una totalidad completa, existe en un cierto instante, infinito actual. El concepto de infinito potencial se centra en un proceso recursivo interminable, puesto que dado un número natural cualquiera, por más grande que sea siempre es posible hallar otro número natural mayor que él, y así sucesivamente. Aristóteles acepta el uso del infinito potencial pero rechaza el del infinito real, para Aristóteles nunca podremos concebir los números naturales como un todo, pero son potencialmente infinitos porque dado un conjunto finito siempre se puede encontrar un conjunto finito mayor que él. Aristóteles en su Libro III de Física afirma que negar la existencia del infinito actual y permitir sólo el infinito potencial no produce ningún obstáculo para los matemáticos. Él consideraba que los matemáticos no necesitaban el infinito y por lo tanto no lo usaban, y que sólo postulan que rechazaba los conjuntos infinitos, las rectas infinitas, y los infinitesimales. Para Aristóteles el geómetra puede considerar segmentos arbitrariamente largos pero no una recta que va hacia el infinito. El pensamiento de Aristóteles acerca del infinito influyó fuertemente en otros matemáticos, tal como una recta puede prolongarse tanto como se desee. Aristóteles fue un firme finitista, que se puede observar en Euclides $\mathrm{y}$ en Arquímedes. 
Euclides demostró que los números primos son potencialmente infinitos. Su demostración consiste en suponer que los números primos son mayores que cualquier cantidad asignada de números primos, es decir, dada una colección finita de números primos debe haber algún número primo mayor que no esté en el grupo.

Arquímedes hacia el siglo III a. C, estuvo muy cerca del moderno infinito matemático. Así es, que anticipándose a Newton y a Leibniz, fue otro precursor del cálculo infinitesimal. Interpretó cien años después de Euclides una de sus definiciones en lo que se conoce como el Axioma de Arquímedes:

Dados dos números positivos a y $\mathrm{b}$, existen números naturales $\mathrm{n}$ y $\mathrm{m}$ tales que na $>b$ y mb $>a$, también llamado Axioma de Eudoxo-Arquímedes (Torres Hernández, 2002, pág. 42).

Este principio expresa que por muy pequeña que sea a y por muy grande que sea $\mathrm{b}$, yuxtaponiendo a consigo misma un número suficiente de veces, superará $\mathrm{a} b$. Esto es una forma de prohibir lo infinitamente pequeño y lo infinitamente grande. Es decir, si a fuese infinitamente pequeña por muchas veces que la sumemos no superará a $\mathrm{b}$. De la misma manera, si $\mathrm{b}$ fuese infinitamente grande no podrá ser superada por ningún múltiplo de a. Como consecuencia se puede decir que las magnitudes que tienen razón se mantienen dentro de unos márgenes "razonables".

Según Gardies. J.L citado por Montesinos Sirera. J.L (1997, pág. 343) en las obras de Arquímedes solamente se nombra la palabra infinito dos veces al comienzo de su obra Arenario, donde trata de refutar el argumento de que el número de granos de arena existentes en el mundo es infinito. La decisión de ocultar dicho término revela las exigencias académicas de la época que se debían cumplir para evitar el infinito considerado por Aristóteles como el "innombrable".

Eudoxo fue el gran matemático griego que supo esquivar y de cierta forma dominar el infinito actual, a él se le atribuye la idea de apartar de las consideraciones matemáticas las cantidades infinitamente pequeñas e infinitamente grandes tal como lo expresa el Axioma de Eudoxo-Arquímedes. Según Rey Pastor \& otros, aunque sea difícil trabajar con el infinito no se puede eludir su uso en Matemática. La magnífica obra realizada por Bolzano (siglo XVIII) y sus continuadores ha consistido en fundar el Análisis infinitesimal sobre el concepto de límite aritmético, en donde sólo interviene el infinito potencial. La aplicación del paso al límite en conceptos fundamentales del Cálculo infinitesimal (derivada e integral) y también la generalidad de las proposiciones matemáticas y las cuestiones sobre el infinito actual y el transfinito justifican la conocida frase de H. Weyl: "La Matemática es la ciencia del infinito" (Rey Pastor, Pi Calleja, \& Trejo, Análisis matemático, 1969, pág. 148).

Según O'Connor J.J y Robertson E.F (2002) hay algunas evidencias recientes que indican que Arquímedes no trató solamente con el infinito potencial. En un 
manuscrito denominado "El Método"2, Arquímedes investigó el número infinito de objetos considerando tres pares de magnitudes infinitas en número y argumentaba que eran respectivamente iguales en número. Esta afirmación sugiere que no todos los objetos infinitos en número son tan iguales. Se cree que no existió ningún otro escrito antiguo donde los objetos infinitos en número se les llame iguales en magnitud a otros objetos infinitos. En un lenguaje más científico se puede decir que Arquímedes se estaba refiriendo al concepto de potencia que posteriormente precisó Cantor: dos conjuntos tienen la misma potencia si se puede establecer una correspondencia biunívoca entre los elementos que pertenecen a uno y otro conjunto. Se puede pensar que Arquímedes podría haberle dado cabida al infinito actual a través de la idea de potencia. Bagni (2001) considera que la idea de que la suma de una serie (en sentido potencial) puede ser finita también estaba presente en Arquímedes ${ }^{3}$. Aunque se cree que Arquímedes conoció los trabajos de Euclides y Aristóteles no emplea en sus obras los mismos métodos que utilizaron ellos. Arquímedes escribía sobre sus investigaciones en informes monográficos y no empleaba los argumentos científicos que usaban ellos.

\section{El germen de los infinitesimales, los indivisibles}

El pensamiento de Aristóteles reinó aproximadamente durante 2000 años porque, según Rey Pastor y Babini, durante la Edad Media no hubo avances en relación al infinito matemático. Las expresiones infinito o infinitésimo actual o potencial que se utilizan hoy en día llevan el sello que les imprimió Aristóteles en aquellos siglos en que la matemática nacía como ciencia (1985, pág. 62). En el origen y desarrollo del Cálculo Infinitesimal podemos citar a muchos estudiosos que expresaron sus ideas en innumerables obras. A partir de los trabajos de los matemáticos griegos, especialmente Arquímedes, se desarrollaron técnicas infinitesimales para calcular áreas y volúmenes. En una primera etapa se introducen los conceptos de indivisibles e infinitésimo. Se pueden citar los indivisibles de Galileo Galilei (1564-1642), Johannes Kepler (1571-1630) y Bonaventura F. Cavalieri (1598-1647) (Díaz García \& Vilela García, 2005, págs. 16-19).

Galileo trata el tema del infinito en su obra Discorsi e dimostrazioni matimatiche intorno a due nuove scienze (1638) y sostiene que el continuo está formado por

2 Tratado rescatado en 1906 en Jerusalén escrito en forma de carta a Eratóstenes. El investigador Heiberg tuvo conocimiento de un palimpsesto, pergamino al que se le había lavado un primer escrito para ser usado con un nuevo texto. El documento se conservó luego de que unos monjes del s XVIII lo hubieran reciclado con textos litúrgicos, sobre el texto de Arquímedes que era copia realizada en el siglo X de una versión anterior. (Gutierrez, 2006)

3 En su obra La cuadratura de la parábola. 
indivisibles partiendo de la misma idea aristotélica: la indivisibilidad indefinida. En oposición al concepto de infinito de Aristóteles que lo concebía como un proceso sin final, Galileo introduce el infinito actual como algo terminado. A partir de sus indivisibles debe aclarar ciertas objeciones mediante el uso de paradojas. Así es como argumenta que aunque se puede establecer una correspondencia biunívoca entre el lado de un cuadrado y su diagonal, la diagonal al tener mayor longitud debería contener más puntos que el lado. Sin embargo, aun estableciendo esa correspondencia no es posible establecer una aritmética con los infinitos y con los indivisibles. Para Galileo los infinitos son incomparables, no se puede establecer que uno es mayor, menor o igual que otro infinito. Un segmento que tenga el doble de longitud que otro no tendrá el doble de infinitos indivisibles. Tampoco es posible comparar un infinito con un finito. Para Galileo las dificultades se debían al tratar con los infinitos y los indivisibles con nuestra mente finita. Se puede decir que Galileo observó que el principio de que el todo es mayor que la parte no vale para conjuntos infinitos. Sin embargo, este posible germen para la posterior teoría de conjuntos desarrollada por Cantor contribuyó más al descrédito del infinito actual que al surgimiento de la teoría. Para algunos no sólo la intuición vuelve implausible la teoría sino que la inabarcabilidad del mismo dificulta la captación de las totalidades infinitas (Sartorio, 2000, pág. 72).

Siguiendo los pasos de Arquímedes, Johannes Kepler en su obra Nova Stereometria Doliorum Vinariorum (1615) expresa de forma sistemática el cálculo de áreas y volúmenes. Dicha obra fue escrita con fines eminentemente prácticos más que teóricos, dado que había sido un año de abundante cosecha de uva. El método de Kepler consistía en seccionar un sólido en un número finito de piezas infinitesimales o sólidos indivisibles de formas y tamaños convenientes de acuerdo a cada problema en particular, la suma de todos esos indivisibles permitía obtener el volumen buscado. Kepler no acuerda con las difíciles demostraciones de Arquímedes por reducción al absurdo, se apoya en el principio de continuidad de Nicolás de Cusa (divinus mihi Cusanus) y realiza sin escrúpulos la operación de paso al límite, identificando una curva con la suma de rectas infinitamente cortas y un área con la suma de rectángulos infinitamente numerosos e infinitamente pequeños.

En relación a Cavalieri ${ }^{4}$ continuando con las ideas de Kepler y Galileo, desarrolla una técnica geométrica para el cálculo de cuadraturas denominada el método de los indivisibles. En este método el área de una figura plana se considera formada por un número infinito de segmentos paralelos equidistantes, cada uno de los cuales se interpreta como un rectángulo infinitamente delgado; y un volumen se lo considera compuesto por un número infinito de áreas de figuras planas paralelas. Es decir, un

4 Fundamentalmente en un tratado que publica en 1635, Geometria Indivisibilibus Continuorum Nova quadam Ratione Promota. 
área es la suma de infinitos segmentos rectos y un volumen es la suma de infinitas superficies planas. A cada uno de estos elementos infinitamente pequeños los llama indivisibles de área y de volumen respectivamente. La técnica de Cavalieri tiene que ver en forma indirecta con la integración y ocupa un lugar intermedio entre las rigurosas demostraciones de Arquímedes y los métodos infinitesimales que surgirán posteriormente. Debido a las críticas recibidas, su método estaba muy lejos de de los fundamentos sobre los que se asienta la geometría euclídea, publica otra obra en 1647 denominada Exercitationes Geometricae en donde expone nuevamente en forma mejorada el método de los indivisibles. Cavalieri adopta los indivisibles de la filosofía escolástica, es decir entes de dimensión menor respecto del continuo del cual forman parte. En esta obra expresa, hablando de los indivisibles, que una línea está hecha de puntos, el plano está hecho de líneas y un sólido de áreas planas. El jesuita Paul Guldin estaba radicalmente en contra del método de los indivisibles de Cavalieri, para él los agregados de infinitas líneas o infinitos planos no se pueden comparar. Según Guldin entre infinito e infinito no hay relación.

En estudios más profundos realizados por Koyré, Cavalieri no usa esta concepción de los indivisibles, ni ninguna otra, para él los indivisibles son solamente una forma de expresarse al referirse a los elementos de dos figuras que compara y mediante técnicas algebraicas le permiten calcular áreas y volúmenes. Aunque el método de Cavalieri recurría en principio al infinito finalmente lo eliminaba. Sin embargo, el método estaba basado en la representación de lo continuo mediante el uso de la intuición geométrica. Su intención era liberarnos del paso al límite con sus imposibilidades lógicas remplazándolo por la intuición geométrica que tiene una legitimidad indiscutida y conserva todas las ventajas de los métodos infinitesimales de Kepler (Koyré, 2007, pág. 328).

El problema que generó la geometría de los indivisibles fue el paso de entidades unidimensionales a entidades bidimensionales al sustituir un elemento lineal por un elemento superficial, se razonaba sobre figuras finitas del espacio considerándolas formadas por elementos infinitamente pequeños.

"A la noción kepleriana de lo infinitamente pequeño, elemento constitutivo del objeto geométrico, que tiene, a pesar de su infinita pequeñez, tantas dimensiones como el objeto en cuestión, opone la de lo indivisible que no es lo infinitamente pequeño y que tiene, rotunda y francamente, una dimensión menos que el objeto estudiado" (Koyré, 2007, pág. 324).

Entre otros, Giles Personne de Roberval (1602-1675) también hizo ciertas contribuciones a los métodos infinitesimales de la época. Roberval le objeta a Cavalieri el querer componer líneas con puntos, superficies con líneas y cuerpos con planos, en vez de emplear los métodos de Kepler.

En 1649 el Colegio Romano rechazó los indivisibles y prohibió su enseñanza en los colegios Jesuitas, y aunque la iglesia quiso silenciar a Galileo poniéndolo 
bajo arresto domiciliario no pudo detener el progreso del cálculo infinitesimal, esto solamente provocó un mayor esfuerzo por parte de los matemáticos para obtener una mayor rigurosidad y así enfrentar las críticas de la época.

Blaise Pascal (1623-1662) fue uno de los principales teóricos que intervino en la crisis producida por el rechazo de los infinitesimales debido, en cierta manera, al ideal escolástico de la deducción. Pascal advirtió que en el método de Cavalieri estaba el recurso de lo infinitamente pequeño y se ocupó de introducir el concepto de infinito por doquier, considerando un infinito tan pequeño como se quiera. Sin embargo, los indivisibles de Pascal no cumplían algunas reglas de la aritmética. Según Brunschvicg, citado por González Gilmas (2004, pág. 98) “....por ejemplo, la proposición que afirma que un indivisible, multiplicado tantas veces como se quiera por una cantidad, está tan alejado de sobrepasar una extensión dada que no puede formar sino uno solo y único indivisible". Siguiendo con este principio Pascal asumió que el indivisible era el cero de la aritmética y esto se oponía al hecho de que un plano estaba compuesto por un número indefinido de líneas. La noción matemática de indivisible refleja la contradicción que había en el pensamiento de Pascal al considerar la división infinita como algo que no puede representarse ni comprenderse pero sin embargo es un principio geométrico que no se puede eludir. Pascal fue el primero en introducir los principios básicos del análisis infinitesimal aunque no manejaba de forma explícita la noción de infinito. Trabajó en forma rigurosa y calculó los límites de las sumas de un número infinitamente grande de cantidades infinitamente pequeñas. Al calcular volúmenes geométricos resolvió los problemas de los diferentes tipos de integración. Su método requería un algoritmo que no pudo obtener y que posteriormente le proporcionaría Leibniz.

A mediados del siglo XVII John Wallis (1616-1703), destacado y original matemático, establece el símbolo actual de infinito $(\infty)$ en su obra Arithmetica infinitorum (1655), también considera el recíproco 1:œ que utiliza para "la nada". La elección de este símbolo se debe al hecho que se puede recorrer la curva indefinidamente. John Wallis también introdujo sistemáticamente el análisis las series infinitas, comenzando por la progresión geométrica. Los primeros trabajos de Newton se basaron en los logros de Wallis, especialmente los que estaban vinculados con el número $\pi$.

En 1658 Fermat identifica una propiedad importante de los enteros positivos que es que no contienen una secuencia descendente infinita. Introduce en 1659 el método de descenso infinito basado en el hecho que si una proposición es verdadera para algún valor entero positivo $\mathrm{n}$, entonces será verdadera para algunos valores enteros positivos menores que n, al no existir una secuencia de descenso infinita en los enteros positivos se caería en una contradicción. Siendo Fermat muy hábil al trabajar con la suma de los términos de una progresión geométrica, obtiene la integración de potencias excepto las de exponente -1 , y utiliza la suma de la serie 
geométrica convergente para calcular cuadraturas. El método que emplea Fermat para calcular cuadraturas puede considerarse como un método de exhaución y consiste en considerar rectángulos infinitesimales inscriptos en la figura a cuadrar cuyas bases deben estar en progresión geométrica. En el método subyacen aspectos esenciales de la integral definida tales como la división del área bajo la curva en elementos de área infinitamente pequeños, aproximación de la suma de esos elementos de área por rectángulos infinitesimales y algo similar a un límite cuando el número de elementos crece indefinidamente mientras se hacen infinitamente pequeños.

Otro protagonista de la época es el jesuita belga Gregorius Saint Vincent (1584-1667) que en una obra voluminosa utiliza la serie geométrica convergente y pretende con ella demostrar la cuadratura del círculo. Con él aparece probablemente por primera vez la distinción entre series convergentes y divergentes. Estudia particularmente series de funciones circulares inversas y al no poder expresar mediante un número finito de términos algebraicos la relación entre el área de un sector circular y la de las poligonales inscriptas o circunscriptas ve la imposibilidad de demostrar la cuadratura del círculo.

\section{Infinito y cálculo infinitesimal}

El desarrollo del cálculo infinitesimal en el siglo XVII ocasionó un avance muy importante en relación al infinito matemático. Los fundadores del cálculo entendieron al mismo como el cálculo de infinitos, o de números infinitamente grandes e infinitamente pequeños, estableciendo de esta manera que el cálculo se ocupa de manipular infinitudes.

Se han señalado numerosas contradicciones ocasionadas al usar los indivisibles, infinitesimales o cantidades infinitamente pequeñas. Las técnicas de cálculo que se fueron desarrollando en forma cada vez más fina no impidieron que subsistieran contradicciones en las fundamentaciones. Particularmente, lo infinitamente pequeño en Newton y Leibniz seguía suscitando problemas de fundamentación. Ambos usaron cantidades infinitamente pequeñas que involucraban el infinito actual pero trataron de hacerlas compatibles con la tradición del infinito potencial aristotélico. Newton lo hizo a través de las primeras y últimas razones y Leibniz intenta una fundamentación teórica a través de la noción de triángulo característico retomando los trabajos de Pascal.

Según O' Connor \& Robertson (2002), Newton priorizó su teoría y rechazó los indivisibles. Su método de las fluxiones, cálculo de derivadas, con su esencia y notación propias resultó ser su contribución más original (Rey Pastor \& Babini, 1985, pág. 82) . Newton presentó su obra en tres escritos. El primero de ellos denominado De Analysi per Aequationes numero terminorum infinitas entrega a su 
maestro Barrow en 1669 y en él se observan conceptos infinitesimales similares a los que usaba el mismo Barrow y ya había usado Fermat. Esta obra, según Pérez González puede considerarse el escrito fundacional del Cálculo. Posteriormente en 1671 realiza una segunda presentación con el nombre Methodus fluxionum et serierum infinitorum que publica recién en 1736, donde considera cantidades variables que van fluyendo con el tiempo y las llama fluentes. También introduce las fluxiones como razones de cambio instantáneas de las fluentes, que son las derivadas respecto del tiempo. El incremento del tiempo y el producto de este incremento por la respectiva fluxión, que Newton denomina "momento", sustituye nuestra diferencial. La notación empleada por Newton y utilizada actualmente en mecánica consiste en indicar las sucesivas fluxiones mediante puntos superpuestos a la fluente. El concepto básico es el de cantidad en movimiento o que fluye continuamente en el tiempo. Las magnitudes no son un agregado de cantidades infinitesimales sino que se generan por el movimiento continuo tal como ocurre en la Naturaleza. Pretendía evitar los problemas matemáticos del continuo, indivisibles e infinitesimales, y llevarlos al mundo físico. Por eso, la idea intuitiva de velocidad instantánea no le pareció necesario tener que definirla. En su última obra Quadrature of Curves (1676) expresa su propósito de abandonar el uso de cantidades infinitesimales. Enuncia su teoría de las "razones primera y última de cantidades evanescentes", anticipándose al concepto matemático de límite. Newton tenía su propia idea de límite: las razones últimas con las que tales cantidades desaparecen son límites a los que tienden a acercarse las razones de cantidades continuamente decrecientes, límites que no pueden traspasarse, ni alcanzarse, antes de que las cantidades disminuyan in infinitum. La fluxión de Newton produjo excelentes resultados pero había mucha cautela a la hora de usar incrementos infinitamente pequeños.

En cuanto a Leibniz, lo novedoso de su cálculo infinitesimal se encuentra en la investigación de lo infinito a partir de las diferencias de diversos órdenes de infinitos y en considerar lo infinitamente pequeño como variación. La nueva noción de Leibniz acerca de lo infinitamente pequeño, poco comprendida por los estudiosos de la época, permitía descender de manera progresiva en un problema cualquiera a las diferenciales de grado infinito (dxdy, ddx, ddy, etc). Por ese entonces, muchos acordaban con Pascal creyendo que sólo se podía acceder a lo infinitamente pequeño a partir de la inmensidad del creador. Se pretendía encontrar un testimonio concreto que mostrara la vinculación entre lo infinitamente pequeño y Dios, por este motivo es que se discutía acerca de la posibilidad de la existencia de los átomos.

Para Leibniz los números infinitos debían rechazarse. Para él el concepto de número aparece solamente cuando se aplica el concepto de cantidad y la cantidad se caracteriza como un entero que posee unidades lo que no es posible en el caso de colecciones infinitas. El infinito debía rechazarse porque el concepto del mismo es lógicamente un absurdo, ¿qué podemos nosotros comprender de los números 
infinitos? Leibniz concibe tres posibilidades: (1) el número de los números enteros, que es el número de los números en la sucesión 1, 2, $3 \ldots$ (2) el número de todos los números, y (3) el número más grande. Cada una de estas nociones para Leibniz es lógicamente un absurdo porque está en conflicto con los principios de la lógica. Las dos primeras acepciones violan el principio de que el entero es mayor que cualquiera de sus partes y por eso Leibniz tiene objeciones con las colecciones infinitas. De la misma manera si no hay números infinitamente grandes tampoco deben considerarse los números infinitamente pequeños, los infinitesimales del cálculo. Leibniz niega los infinitesimales.

Ni Newton ni Leibniz resolvieron los problemas lógicos de fundamentación. La mayor crítica recibida fue por parte de Berkeley (1605-1753), obispo de Cloyne en Irlanda, quien evidencia en un ensayo la falta de rigurosidad del cálculo y sugiere ciertas correcciones. Su famosa cita expresa en forma abreviada las objeciones planteadas: “¿Y qué son estas fluxiones? Las velocidades de incrementos evanescentes. ¿Y qué son los mismos incrementos evanescentes? No son más que cantidades finitas, no cantidades infinitamente pequeñas, ni nada de eso. ¿No podríamos llamarlos fantasmas o cantidades huidas?" (O' Connor \& Robertson, 2002). Según Recalde (2004, pág. 54), Berkeley ${ }^{5}$ planteaba reparos sobre el uso de aquellos aspectos ligados a la palabra infinito, específicamente al infinito en acto. Para él había ciertas inconsistencias con los infinitesimales al no cumplir el principio de Arquímedes. Consideraba que los matemáticos no eran coherentes al trabajar con los infinitesimales dado que al comienzo los usaban en los denominadores por ser diferentes de cero, pero luego si aparecían como sumandos eran despreciados. Entre 1700 y 1706 fueron famosos los debates en la Académie des Sciences de París sobre la validez de los procesos del nuevo cálculo. El Marqués de L'Hôpital 'intentó formalizar el concepto intuitivo y la operatividad de los infinitesimales lo que ocasionó sesiones de debates y discusiones, y aunque hizo una gran contribución al cálculo al querer popularizarlo no pudo evitar ser objeto de las críticas de Berkeley.

Leibniz dudaba de su propia rigurosidad tal como lo manifiesta en su testamento intelectual escrito en septiembre de 1716 poco antes de morir: "En cuanto al cálculo de los infinitesimales yo no estoy del todo satisfecho con las expresiones del señor Herman en su respuesta al señor Nieuwentijt, ni de otros amigos. También M. Naudé tiene razón de hacer oposición. Cuando discutía en Francia con el abat Gallois, el padre Gouge y otros, les manifesté que no creía que hubiera magnitudes

5 En su obra The Analyst; or, a Discourse Addressed to an Infidel Mathematician. Wherein It is examined whether the Object, Principles, and Inferences of the modern Analysis are more distinctly conceived, or more evidently deduced, than Religious Mysteries and Points of Faith. By George Berkeley (1734).

6 En su publicación Analyse des infiniment petits pour l'intelligence des lignes courbes (Análisis de los infinitésimos para el estudio de las líneas curvas). 
verdaderamente infinitas ni verdaderamente infinitesimales: que sólo eran ficciones pero ficciones útiles para abreviar y hablar universalmente. Pero como el señor Marqués de L'Hospital creía que por ello yo traicionaba la causa, me rogaron que no dijera nada, aparte de lo que había dicho en un lugar de las Actas de Leipzig; con placer accedí a ese ruego" (Leibniz citado por Recalde (2004, pág. 54)).

Newton arribó a sus descubrimientos en la mitad del año 1660, aproximadamente 10 años antes que Leibniz, pero realizó sus publicaciones más tarde. Sin embargo, entre ellos y entre sus discípulos hubo un furioso debate acerca de quién de ellos es el primero y merecedor del gran crédito.

Posteriormente, el matemático nacido en Basilea Leonhard Euler (1707-1783) publicó un libro de texto Introductio sobre cálculo que tuvo también considerable influencia para los matemáticos de la época. Es un trabajo magistral organizado de una forma en que los matemáticos han coincidido en considerar bella que puede comprenderse en términos puramente analíticos y no geométricos. En carta a su amigo Goldbach en 1744 le escribe: " Después de haberme hecho el plan de un tratado completo sobre el análisis de los infinitos me di cuenta de que tenían que precederle muchas cosas que propiamente no están incluidas en él ni se encuentran apenas tratadas en ninguna parte; y de ellas ha salido esta obra como pródromo al análisis de los infinitos" (Durán, 2003). En esta obra no hay cálculo diferencial ni integral sino que es una introducción al cálculo con los infinitos: se estudian funciones elementales por medio de procesos infinitos, se desarrollan en series, y por primera vez en la historia de la matemática se desarrollan algunas funciones en productos infinitos. Para obtener los desarrollos en serie Euler utilizó como herramienta cantidades infinitamente grandes e infinitamente pequeñas. Son los infinitos los que le permiten poner en evidencia la estructura interna de las funciones. En el análisis actual esas cantidades infinitamente grandes y pequeñas han desaparecido. Según Moore (1990) Euler usa los infinitesimales de manera poco satisfactoria, aunque algunos consideran que el análisis matemático usando cantidades infinitesimales es tan riguroso como el que se hace usando límites.

La figura de transición parece ser Cauchy (1789-1857) al proporcionar la primera salida conceptual al problema de los infinitesimales. Cauchy, es quien percibe la necesidad de despegar las nociones de intuiciones ancladas en registros visuales, y su definición de infinitésimo, aunque lejos del registro formal actual, es ya diferente de las precedentes, aunque aún permanece en registros verbales. En 1821 escribe su Analyse algébrique (Curso de Análisis) en donde institucionaliza el concepto de límite y a través del límite incorpora las cantidades infinitamente pequeñas e infinitamente grandes. El capítulo 2 del Curso de Análisis sobre las funciones continuas comienza con un pequeño tratado sobre las cantidades infinitamente pequeñas e infinitamente grandes. Deja de considerar los infinitésimos con valor fijo, no nulo e indeterminado. Sus infinitamente pequeños son cantidades variables que tienen al cero como límite. 
Su aporte más importante consistió en haber establecido con precisión definiciones, delimitar el campo de validez de las fórmulas y eliminar extensiones ilegítimas. Los trabajos de Cauchy conformaron el marco necesario para la completa fundamentación del análisis. Sin embargo, es Weierstrass (1815-1897) quien establece la moderna definición de límite en el lenguaje épsilon-delta, proveyendo una vía de acceso al infinitésimo potencial. Se puede decir que con Cauchy y Weierstrass se reconsideró el método de exhaución y comenzaron a darle a la Matemática un cimiento estable.

Bolzano (1781-1848) publica en 1817 Rein analytische Beweis (Demostración analítica pura) en este libro intenta liberar al cálculo del concepto infinitesimal. Waldegg (1996) señala que la concepción de Bolzano del infinito matemático produce un cambio significativo porque modifica toda una tradición milenaria. En cuanto al desarrollo del concepto de infinito en la publicación Paradoxes of the infinite de 1840 reivindica la existencia del infinito actual utilizando la idea de conjunto y reconoce que la diferencia entre conjuntos finitos e infinitos es la posibilidad de estar en correspondencia con una parte propia, como ya lo había observado Galileo. Bolzano considera dos principios sobre los que debe edificarse el concepto de infinito: (a) los conjuntos infinitos se pueden determinar mediante la axiomatización de la teoría de conjuntos: la extensión y la comprensión, (b) los conjuntos infinitos no tienen todos el mismo tamaño. A partir de estos principios pretende comparar los conjuntos infinitos, el tipo de comparación que realiza Bolzano es diferente al que hará Cantor, su criterio de comparación se basa en la relación de inclusión, mientras que Cantor lo hará mediante la correspondencia uno a uno.

Bolzano le asignó un nuevo rol al infinito evidenciando la presencia de un nuevo nivel de representación, abandona la idea de un conjunto que resulta de un proceso constructivo agregando elementos. Sin embargo, aunque transformó el infinito en un objeto no pudo aritmetizarlo.

No se puede prescindir del infinito en Matemática, la gran obra de Bolzano y sus continuadores consistió en fundar el Análisis infinitesimal sobre el concepto de límite aritmético donde solamente interviene el infinito potencial.

La aritmetización del análisis se produjo en la década del 1870. Se disiparon las dudas metafísicas y se lograron los fundamentos del análisis sobre bases aritméticas claras y firmes. La esencia de todo este proceso consistió en incorporar a las operaciones aritméticas una nueva operación "el paso al límite". Esta herramienta le otorgó rigor a los métodos infinitesimales iniciados por Newton y Leibniz. La novedosa aplicación de paso al límite ya sea mediante el infinito numerable o mediante el infinito continuo le dio al análisis clásico nuevos algoritmos: las series y las integrales son combinaciones del paso al límite con la suma, el producto infinito lo es con la multiplicación, la derivada con la división; por eso aplicado a procesos algebraicos o funcionales le daban surgimiento a nuevos algoritmos.

En 1877 Cantor (1845 -1918) determina que se puede establecer una 
correspondencia biunívoca entre un cuadrado y uno de sus lados y posteriormente establece el concepto de potencia. Leibniz tuvo la intuición de la existencia de infinitos de distintos órdenes sugerido por el tipo de funciones a las que le calculó su derivada.

Dieudonné, citado por Hernández expresa: "Contrariamente a una opinión muy extendida, no hay que creer que los analistas del siglo XVIII sean indiferentes a las cuestiones de aproximación y de convergencia, casi todos ellos se interesan por el cálculo numérico", para concluir "Las acusaciones de falta de rigor que hubieron de sufrir los analistas del siglo XVIII por parte de sus sucesores viene sobre todo, de hecho, de la dificultad que tuvieron para definir de modo preciso las nociones básicas del cálculo infinitesimal de las que tenían a menudo una buena concepción intuitiva, y en marcar la distinción, a veces sutil, entre nociones aparentemente próximas; en el siglo XIX fueron condenados de modo demasiado expeditivo teniendo en cuenta solo lo incorrecto de su lenguaje, sin examinar demasiado el contexto" (Hernández, 1997, págs. 197, 198).

Este recorrido histórico pone de manifiesto las fuertes resistencias que el concepto del infinito actual suscitaron entre eminentes matemáticos, incluyendo a Poincaré y Kronecker, probando la carga de abstracción y flexibilidad que tal objeto demanda. Para Poincaré, según de Lorenzo (2005, pág. 23), el término infinito es muy confuso, al decir todos no se distingue entre el infinito actual o potencial, el único infinito es un infinito en devenir, el potencial y por eso carece de sentido dar una definición en términos de sus elementos como si estuvieran dados en acto. Kronecker (Solaeche Galera, 1995) insiste en descartar el infinito edificando toda la matemática a partir de los números naturales, es un partidario del finitismo que rechaza el infinito "...futilidad perniciosa heredada de filosofías anticuadas y teologías confusas, pudiendo llegar sin él tan lejos como se quiera...".

Con Cantor y Dedekind se estableció la fundamentación de los números reales, ambos formalizaron y generalizaron el proceso de aproximación de algunos irracionales. Cantor lo hace a través de la noción de sucesión fundamental y Dedekind a partir del concepto de cortadura. Las construcciones de ambos son equivalentes y cumplen con el principio de Arquímedes.

Entre los años 1879 y 1897 Cantor establece los conceptos básicos para instaurar los ordinales y cardinales transfinitos. Los transfinitos, con una identidad numérica, aparecen por primera vez en 1882 en un manuscrito denominado Grundalagen einer allgemeinen Mannigfaltigkeitslehre (Fundamentos de una teoría general de conjuntos), la misma estaba estructurada sobre la noción del infinito actual. Su éxito fue negar la afirmación obvia de que el todo es mayor que cualquiera de sus partes. Así es que hay tantos números naturales como números pares, tantos números fraccionarios como números naturales, siendo este número el número cardinal Alef cero. Además evidenció la existencia de un número cardinal más elevado, el número de puntos de una recta, igual al número de puntos de un cuadrado, de un cubo o 
del espacio entero. Científicamente se puede decir que Cantor precisó el concepto de potencia de una colección infinita de objetos, teniendo dos conjuntos la misma potencia si es posible establecer una correspondencia biunívoca entre los elementos que pertenecen a uno y otro conjunto. Uno de los problemas que más preocupó a Cantor fue determinar el cardinal transfinito que le corresponde a la potencia del continuo. Su determinación corresponde al primero de los problemas planteados en 1900 por David Hilbert (1862-1943) en el Congreso de París, en su discurso se refirió entre otros temas a la hipótesis del continuo, la hipótesis del orden total y la consistencia del sistema de los números reales. Hilbert consideraba que todo problema matemático bien planteado tiene solución, su pensamiento se caracterizó por destacar la importancia de los problemas en la investigación matemática, sino se tiene un problema definido en mente es probable que se investigue en vano.

Desde el comienzo Cantor planteó las diferencias entre el infinito actual y el infinito potencial. Él rechazaba las cantidades infinitamente pequeñas porque parecían contradecir la concepción misma del infinito actual. Cantor en carta a Weierstrass (Recalde, 2004, pág. 66) le manifiesta: "Los números lineales, no cero, (resumiendo, números los cuales pueden ser pensados como longitudes de una línea recta, acotados y continuos), los cuales serán más pequeños que cualquier número arbitrario, no existen, esto es, ellos contradicen el concepto de número lineal". Según Cantor los infinitesimales no tienen mucha importancia porque carecen de una estructura propia como un cuerpo teórico matemático. Posteriormente los trabajos de Robinson demostrarían otra cosa.

A mediados del siglo XX el matemático Abraham Robinson inicia el análisis no estándar usando la teoría de modelos en la fundamentación de los infinitesimales. Sin embargo, algunos elementos de su teoría ya habían sido introducidos por el lógico noruego Thoralf Skolem.

La noción ontológica de número ha ido variando de acuerdo a los cambios históricos conceptuales, la lógica nos proporciona un universo más amplio y acepta algunos objetos que habían sido descartados, así es como Robinson con su modelo no estándar de los números reales donde los números infinitos tienen sentido, nos insinúa que la legalidad matemática no es de ninguna manera absoluta.

Claudia López 


\section{Referencias}

Alsina, C. (2002). Why the Professor mus be a Stimulating. En D. Holton (ed.), The Teaching and Learning of Mathematics at University Level. An ICMI Study (Primera ed., págs. 3-12). Dordrecht: Kluwer Academic Publishers.

Anglin, W. (1994). Mathematics: a concise history and philosophy. SpringerVerlag New York Inc.

Apostol, T. (1984). Calculus (Vol. I). Barcelona, España: Reverté S. A.

Arboleda, L. C. (Diciembre de 2002). Los tratados franceses en la enseñanza del análisis en Colombia (1851-1951): Sturm, Humbert y los otros. Recuperado el 21 de Agosto de 2011, de Universidad del Valle. Colombia: http://www. accefyn.org.co/revista/Vol_26/101/533-543.pdf

Azcárate Giménez, C. \&. (2003). Sobre la Investigación en Didáctica del Análisis Matemático. Boletín de la Asociación Matemática Venezolana, X(2), 135-149.

Bagni, G. T. (2001). Infinito e infintesimo potenziale e attuale: una sfida per la Scuola Secondaria Superiore. Bolletino dei Docenti di Matematica, II(42), 9-20.

De Lorenzo, J. (2005). Poincaré, pensador de la matemática. Recuperado el 1 de Octubrede2011, dehttp://upcommons.upc.edu/video/bitstream/2099.2/247/6/247_ Article.pdf

Díaz García, L., \& Vilela García, M. Á. (20 de Enero de 2005). El infinito matemático. Recuperado el 15 de julio de 2011, de Universidad de La Laguna. Canarias: http:// www.miguev.net/blog/wp-content/uploads/2005/01/El_Infinito_Matematico.pdf

Durán, A. J. (2003). Euler y los infinitos (grandes y pequeños). Recuperado el 10 de Enero de 2013, de http://upcommons.upc.edu/e-prints/bitstream/2117/1985/1/ mblanco_paper_icehve.pdf

Eggers Lan, C. (1995). El nacimiento de la matemática en Grecia. Buenos Aires: Universitaria de Buenos Aires. (EUDEBA).

Festa, E. (s.f.). Atomismo y continuo en el origen de la ciencia moderna. Recuperado el 26 de ocubre de 2012, de Centro Alexandre Koyré. París: http://www.gobiernodecanarias.org/educacion/3/usrn/fundoro/archivos\%20 adjuntos/publicaciones/actas/act_9_pdf_web/05_Egidio_Festa.pdf

Garbin, S., \& Azcárate, C. (2002). Infinito actual e inconsistencias: acerca de las incoherencias en los esquemas conceptuales de alumnos de 16-17 años. Enseñanza de las Ciencias, 20 (1), 87-113. 
Garden R., Lie, S., Robitaille, D., AngellL, C., Martin, M., Mullis, I., Foy, P., Arora, A. (2006). TIMSS advanced 2008 assessment frameworks. Boston: TIMSS \& PIRL INTERNATIONAL STUDY CENTER. Recuperado el 3 de abril de 2011, de http://timss.bc.edu/PDF/TIMSS_Advanced_AF.pdf

Gonzáles Gilmas, O. (2004). El cálculo infinitesimal Leibniciano: Una síntesis de las perspectivas de Brunschvicg e Ishiguro. Signos Filosóficos, VI(11), 97-120.

González Urbaneja, P. M. (Diciembre de 2008). La solución de Eudoxo a la crisis de los inconmensurables. Sigma(33), 101-130. Recuperado el 15 de Julio de 2011, de http://www.hezkuntza.ejgv.euskadi.net/r43-573/es/contenidos/informacion/ dia6_sigma/es_sigma/adjuntos/sigma_33/8_solucion_eudoxo_33.pdf

González, K. G. (Enero-Abril de 2003). Origen, Renacimiento y Destierro de los infinitesimales. Revista Educación y Pedagogía, XV(35), 29-36. Recuperado el 13 de Julio de 2011, de http://revinut.udea.edu.co/index.php/revistaeyp/ article/viewFile/5941/5351

González, S. V. (2003). Platón, Teeteto. Madrid: Biblioteca Nueva, S. L.

Hernández, J. (Noviembre de 1997). El rigor en el Análisis del siglo XIX: de Cauchy a Weierstrass. Recuperado el 20 de Noviembre de 2012, de Departamento de Matemática. Universidad Autónoma de Madrid: http:// www.gobiernodecanarias.org/educacion/3/Usrn/fundoro/web_fcohc/005_ publicaciones/seminario/certidumbre.htm

Korn, F., Lazarsfeld, P., Barton, A., \& Menzel, H. (1969). Conceptos y variables en la investigación social. Selección de Francis Korn y Manuel Mora y Araujo. Buenos Aires: Nueva Visión.

Koyré, A. (2007). Estudios de historia del pensamiento cientifico. México: siglo xxi de españa editores, s.a.

Krippendorff, K. (1990). Metodología de análisis de contenido. Teoría y práctica. (L. Wolfson, Trad.) Barcelona: Paidós.

Le Lionnais, F. y. (1976). Las grandes corrientes del pensamiento matemático. Buenos Aires: EUDEBA.

López Pellicer, M. (2005). La estructura racional del pensamiento matemático. El infinito matemático. Recuperado el 14 de Julio de 2011, de Real Academia de Ciencias: http://www.rac.es/ficheros/doc/00352.pdf

Lupiannez, J. (2009). Nuevas Visiones en la Matemática: Vieta y Wallis. Recuperado el 25 de Julio de 2011, de Nuevos Acercamientos a la Historia de la Matemática a través de la Calculadora TI-92: http://cumbia.ath.cx:591/pna/ 
Archivos/LupiannezJ00-2710.PDF

Martín, C. M. (Enero de 1998). Eudoxo y la Matemática. Recuperado el 13 de Julio de 2011, de Universidad de La Laguna. Canarias: http:/www. gobiernodecanarias.org/educacion/3/usrn/fundoro/archivos\%20adjuntos/ publicaciones/actas/actas_6_7_pdf/Act.VI-VII_C011_txi_w.pdf

Montesinos Sirera, J. L. (1997). Arquímedes y la medida del círculo. Recuperado el 14 de Julio de 2011, de Universidad de la Laguna. Canarias: http://www. gobiernodecanarias.org/educacion/3/usrn/fundoro/archivos\%20adjuntos/ publicaciones/actas/actas_6_7_pdf/Act.VI-VII_C016_txi_w.pdf

Montoro, V., \& Scheuer, N. (2004). Pensando el infinito. Concepciones de estudiantes universitarios. Centro Regional Bariloche. Universidad Nacional del Comahue. República Argentina. Recuperado el mayo de 2010

Moore, A. W. (1990). The infinite. London and New York: Routledge. Taylor \& Francis Group.

Nápoles Valdes, J. E. (2003). Paradojas y Fundamentos de la Matemática. Historia y Pedagogía. Recuperado el 14 de Julio de 2011, de http://cabierta. uchile.cl/revista/27/articulos/pdf/paper3.pdf

O' Connor, J. J., \& Robertson, E. F. (Febrero de 2002). Mac Tutor History of Mathematics. Recuperado el 2 de Mayo de 2010, de History Topic: Infinity: http://www-history.mcs.st-and.ac.uk/HistTopics/Infinity.html

Ortiz, J. R. (1994). El concepto de infinito. Boletín de la Asociación Matemática Venezolana. I(2), 59-81.

Pérez de Laborda, A. (2005). Estudios filosóficos de historia de la ciencia. (E. E. A, Ed.) Recuperado el 19 de Septiembre de 2011, de http://www.apl.name/ Alfonso/Historia\%20de \%201a\%20ciencia.pdf

Pérez González, J. (2008). Orígenes del Cálculo. Recuperado el Julio 31 de 2011, de Historia de la Matemática. Universidad de Granada: http://www.ugr. es/ dpto_am/docencia/Apuntes/Origenes_del_Calculo.pdf

Recalde, L. C. (junio de 2004). La lógica de los números infinitos: un acercamiento histórico. Enseñanza Universitaria, XII(1), 51-72.

Rescher, N. (Enero de 1955). Leibniz' conception of quantity, number, and infinity. The Philosophical Review, 64(1), 108-114.

Rey Pastor, J., \& Babini, J. (1984). Historia de la Matemática. De la Antigüedad a la Baja Edad Media (Primera edición ed., Vol. I). Barcelona, España: Gedisa. 
Rey Pastor, J., \& Babini, J. (1985). Historia de la Matemática. Del Renacimiento a la Actualidad (Primera Edición ed., Vol. II). Barcelona, España: Gedisa.

Rey Pastor, J., Pi Calleja, P., \& Trejo, C. A. (1969). Análisis matemático (Vol. I). Buenos Aires, Argentina: Kapelusz.

Sartorio, A. C. (2000). Conjuntos e Infinitos (Primera edición ed.). Buenos Aires: Eudeba.

Sellés García, M. (2001). La teoria de indivisibles de Galileo y su geometrización del movimiento. Recuperado el 14 de Julio de 2011, de http://www. gobiernodecanarias.org/educacion/3/usrn/fundoro/archivos\%20adjuntos/ publicaciones/largo_campo/cap_03_09_Selles.pdf

Solaeche Galera, M. C. (1995). La controversia entre L. Kronecker y G. Cantor acerca del infinito. Divulgaciones Matemáticas 3 (1/2), 115-120.

Tall, D. (1980b). The notion of infinite measuring number and its relevance in the intuition of infinity. Published in educational studies in mathematics, 11, 271284, 1980. Recuperado el 23 de abril de 2010, de The University of Warwick. David Tall, Professor in Mathematical Thinking: http://www.warwick.ac.wk/ staff/David. Tall/themes/limits-infinity.html

Tall, D. (2001). Natural and formal infinities. Recuperado el 23 de abril de 2010, de The University of Warwick. David Tall, Professor in Mathematical Thinking: http://www.warwick.ac.wk/staff/David. Tall/themes/limits-infinity.html

Toledo Prats, S. (2003). El significado filosófico de las matemáticas en la cultura griega. (M. P. Wissenschaftsgeschichte, Ed.) Symposium Arquímedes, 73-86. Recuperado el 14 de Julio de 2011, de http://www.gobiernodecanarias.org/ educacion/3/usrn/fundoro/archivos\%20adjuntos/publicaciones/Arquimedes/ Toledo_FilosofiaMatematicaGriega.pdf

Torres Hernández, E. (2002). Eudoxo, Arquímedes y el límite de una sucesión. Recuperado el 10 de Juliio de 2011, de Universidad Autónoma de México: http://albertofest.matcuer.unam.mx/Misc35/Eudoxo.pdf

Waldegg, G. (Enero-Junio de 1996). Identificación de obstáculos didácticos en el estudio del infinito actual. Revista Mexicana de Investigación Educativa, I(1), 107-122. Recuperado el 19 de Marzo de 2012, de Revista Mexicana de Investigación educativa. Vol 1, núm 1, pp:107-122: http://redalyc.uaemex.mx/ pdf/140/14000108.pdf

Waldegg, G. (2008). Homenaje a una trayectoria. Compiladora Irma Fuenlabrada. Recuperado el 15 de Enero de 2013, de Universidad Pedagógica Nacional. México: www.upn.mx 